\title{
Every ten minutes a child has a rotten tooth removed
}

New data published by Public Health England (PHE) on the same day that the Soft Drinks Industry Levy came into effect, 6 April, show that a child in England has a tooth removed in hospital every ten minutes due to preventable decay.

PHE's Change4Life campaign is reminding parents that sugary drinks - including juice drinks, energy drinks, cola and other fizzy drinks - are one of the main sources of sugar in children's diet.

Tooth extraction remains the most common reason for hospital admissions in 5-9-year-olds. Figures show around 141 children a day - some just a year old - are having teeth removed. This means around 60,000 days are missed from school during the year, as well as causing problems with eating, sleeping and even smiling.

Dr Sandra White, Dental Lead for Public Health England, said: 'It's upsetting to see so many children admitted to hospital with tooth decay, but swapping out sugary drinks could be an easy win for busy families.

'Parents can also help prevent decay by making sure their children's teeth are brushed

twice a day with fluoride toothpaste and reducing how much sugar they're eating and drinking.'

For more information on the Change4Life campaign, visit https:// www.nhs.uk/change4life/food-facts/healthier-snacks-for-kids/ lower-sugar-drinks-for-kids-stop-tooth-

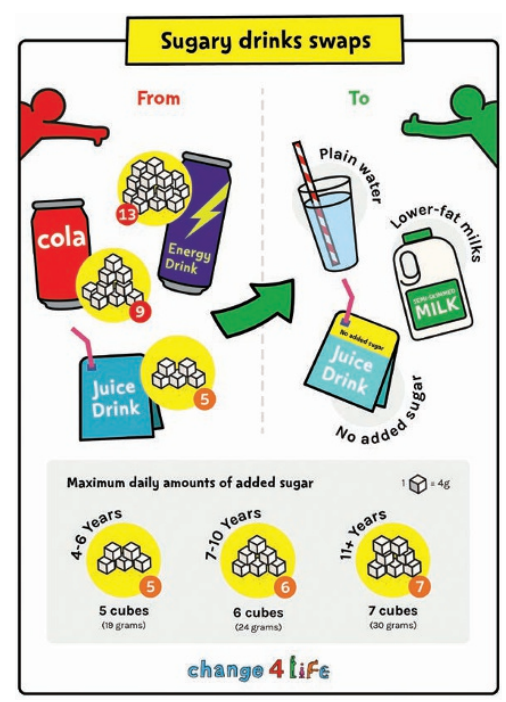

The 'Sugary drinks swaps' suggestions that are part of PHE's Change4Life campaign
decay\#4ScekU62Pw0QbYMY.97.

The British Dental Association (BDA) has accused English authorities of resting on their laurels in the face of the 'decay epidemic'.

Henrik Overgaard-Nielsen, the BDA's chair of General Dental Practice, said: 'In the face of a tooth decay epidemic the official response remains woefully inadequate.

'While devolved governments have rolled up their sleeves [with Scotland's Childsmile and Wales' Designed to Smile proving successful], authorities in England have chosen to rest on their laurels. The result is an oral health gap that shows no signs of closing.

'To date not a single child has seen any benefit from the government's unfunded and unambitious plans to act on decay. [PHE's] figures underline the need for concerted national action. 